\title{
Auditoria interna do sistema de gestão da qualidade na distribuição de produtos
}

\section{para saúde: estudo de caso}

\author{
The internal audit of the quality management system in a medical devices distributor: a case study \\ Auditoria interna del sistema de gestíon de la calidad en la distribución de productos sanitarios:
}

caso de estudio

Recebido: 28/11/2021 | Revisado: 03/12/2021 | Aceito: 03/12/2021 | Publicado: 04/12/2021

\author{
Alex Sander Lopes da Silva \\ ORCID: https://orcid.org/0000-0002-5918-1366 \\ Universidade do Estado da Bahia, Brasil \\ E-mail: sanderlopes@gmail.com \\ Jucimar Casimiro de Andrade \\ ORCID: https://orcid.org/0000-0001-5539-4498 \\ Centro Universitário Maurício de Nassau, Brasil \\ E-mail: jucimar.casimiro@ sereducacional.com \\ Clícia Maria de Jesus Benevides \\ ORCID: https://orcid.org/0000-0002-7214-1318 \\ Universidade do Estado da Bahia, Brasil \\ E-mail: cbenevides@uneb.br
}

\begin{abstract}
Resumo
O mercado de produtos para saúde é um setor no qual as agências regulatórias exercem grande pressão sobre o controle e a gestão da qualidade, a fim de prevenir danos à saúde individual e coletiva. Assim, as auditorias internas (AUDIN) são apontadas como fundamentais para o monitoramento e o aprimoramento da qualidade de produtos, serviços e sistemas de gestão da qualidade (SGQ). O presente estudo, realizado entre janeiro e fevereiro de 2021, visou investigar o impacto da AUDIN na manutenção e no aprimoramento do SGQ de uma empresa distribuidora de produtos para saúde localizada em Salvador/BA. Observou-se que o SGQ do caso em análise seguia os parâmetros definidos pela RDC 16/2013, tendo os resultados das AUDIN como norteadores para promoção do ciclo PDCA. Foram identificadas não-conformidades (NC) pontuais em $8 \%$ dos requisitos auditados, todos com desenvolvimento de plano de ação, levantamento de medidas corretivas e preventivas e avaliação de eficácia, apresentando eficácia de $60 \%$ e $80 \%$, após sete e vinte dias, respectivamente, de onde se conclui que a AUDIN apresentou grande efetividade para a deteç̧ão e correção de NC no SGQ, auxiliando na prevenção de danos à saúde individual e coletiva dos usuários.
\end{abstract}

Palavras-chave: Auditoria interna; Gestão da qualidade; Saúde coletiva.

\begin{abstract}
The medical devices market is a sector in which regulatory agencies exert great pressure on quality control and management, in order to prevent damage to individual and collective health. Thus, internal audits are seen as fundamental for monitoring and improving the quality of products, services and quality management systems (QMS). The present study, carried out between January and February 2021, aimed to investigate the impact of AUDIN on the maintenance and improvement of the QMS of a healthcare products distributor located in Salvador/BA. It was observed that the QMS of the case under analysis follows the parameters defined by RDC 16/2013, with the AUDIN results as guidelines for promoting the PDCA cycle. Non-conformities (NC) were identified in $8 \%$ of the audited requirements, all with the development of an action plan, survey of corrective and preventive measures and evaluation of effectiveness, showing $60 \%$ and $80 \%$ effectiveness, after seven and twenty days, respectively, from which it is concluded that AUDIN showed great effectiveness for the detection and correction of NC in the QMS, helping to prevent damage to the individual and collective health of users.
\end{abstract}

Keywords: Internal audit; Quality management; Collective health.

\section{Resumen}

O mercado de produtos sanitários é um setor em que os organismos reguladores exercem uma grande pressão sobre o controle e a gestão da qualidade, com o fim de prevenir daños para a saúde individual e coletiva. Assim, as auditorías internas (AUDIN) são consideradas fundamentais para monitorar e melhorar a qualidade dos produtos, serviços e sistemas de gestão da qualidade (SGC). O presente estúdio, realizado entre enero e fevereiro de 2021, tuvo como objetivo investigar o impacto de AUDIN na manutenção e mejora do SGC de um distribuidor de produtos sanitários ubicado em Salvador / BA. Veja que o SGC do caso na análise siguió os parâmetros definidos por el RDC 16/2013, 
com os resultados do AUDIN como pautas para impulsar o ciclo PDCA. Se identificação de não conformidades (NC) em $8 \%$ dos requisitos auditados, todo ello com o desarrollo de um plano de ação, relevamiento de medidas corretivas e preventivas e avaliação de efectividad, mostrando uma efectividad del $60 \%$ y $80 \%$, after the siete and veinte. Aí, respectivamente, de los cuales se concluye que o AUDIN mostrou gran efectividad para a detecção e correcção de NC no SGC, ayudando a prevenir daños a la salud individual y colectiva de los usuarios.

Palabras clave: Auditoria interna; Gestión de la calidad; Salud pública.

\section{Introdução}

Produtos para Saúde, produtos médicos, produtos odonto-médico-hospitalares, correlatos e OPME (Órteses, Próteses e Materiais Especiais) são nomenclaturas popularmente utilizadas no Brasil para definir produtos que, internacionalmente, são tratados como Dispositivos Médicos (Medical Devices ou MD). A Agência Nacional de Vigilância Sanitária (ANVISA), na RDC 185 de 22 de outubro de 2001, classifica Produtos para Saúde ou Produto Médico como:

(...) todos os produtos destinados à prevenção, diagnóstico, tratamento, reabilitação ou anticoncepção que não utilizam meio farmacológico, imunológico ou metabólico para realizar sua principal função em seres humanos, podendo, entretanto, ser auxiliados em suas funções por tais meios. (ANVISA, 2001)

Com a tendência de aumento na expectativa de vida e o acelerado desenvolvimento de novos produtos para saúde, o mercado de dispositivos médicos tem crescido significativamente. Como os gastos com dispositivos médicos no Brasil estão percentualmente abaixo dos valores verificados em muitos países, percebe-se que ainda há uma grande demanda reprimida, provavelmente devida ao fato de uma parcela significativa da população ainda não ter acesso a tratamentos mais onerosos, com tecnologia de ponta (Marrone, 2015).

Tratando-se de um mercado financeiramente promissor para as empresas e com enorme impacto social, por estar diretamente ligado à manutenção e à preservação da vida, é de se esperar que a qualidade seja um dos pilares para norteá-lo. A preocupação com a qualidade dos MD não é tema apenas das empresas produtoras, importadoras e distribuidoras e das organizações internacionais de padronização, como a International Organization for Standardization (ISO), mas principalmente de órgãos governamentais de regulação, como o Food and Drug Administration (FDA) nos EUA, o Comitê Europeu de Normalização ou Comissão Europeia (CEN ou CE) e, no Brasil, a Agência Nacional de Vigilância Sanitária (ANVISA), devido a sua elevada importância para a saúde individual e coletiva (Martins, 2013).

Deste modo, diversos aspectos e normatizações, no que tange à gestão da qualidade nestas empresas, além de caráter técnico, recebem um caráter de ordem legal, sendo regidos por legislações sanitárias específicas, de forma a garantir a adequação ao uso, com segurança, eficácia e melhoria contínua (Martins, 2013; Guerra-Bretaña \& Florez-Rendón, 2018; Lingg et al., 2018), sendo esta última caracterizada pela buca de melhores resultados e aumento no nível d e desempenho dos processos (Santos, et al., 2020).

A empresa utilizada como estudo de caso é uma empresa importadora e distribuidora de produtos para saúde, localizada na cidade de Salvador/BA. Atuante no ramo há cerca de quinze anos, a empresa distribui produtos para saúde fabricados no sudeste asiático, Europa, Estados Unidos e Brasil, com foco em implantes ortopédicos confeccionados em titânio para cirurgias bucomaxilofaciais, comercializando também diversos outros itens de Classe de Risco I, II III e IV, segundo classificação da ANVISA (ANVISA, 2011). Como toda empresa do ramo de produtos para saúde em nosso país, a "empresa caso" necessitou desenvolver um Sistema de Gestão da Qualidade (SGQ) que foi inspecionado pela vigilância sanitária para obtenção das autorizações de funcionamento e licenciamento sanitário e mantém seu constante monitoramento e melhoramento, a partir da análise dos dados obtidos através dos registros operacionais.

Sabe-se que criar e gerir um SGQ em uma empresa não é uma tarefa simples. Apesar de existirem diversos programas e ferramentas, um programa pode lograr grandes êxitos em um tipo de empresa e não ter o mesmo sucesso em 
outros. Além disto, há normas específicas quanto a pontos chave no SGQ que devem ser seguidos por força de lei, a depender do ramo de atividade, como é o caso da RDC 16/2013 para fabricantes, importadores e distribuidores de produtos para saúde localizados no Brasil ou que comercializem seus produtos no país (ANVISA, 2013). Deste modo, entende-se que estudos de caso sobre o SGQ de empresas do setor podem ser extremamente úteis para difundir experiências, auxiliar na elevação do padrão da qualidade geral e, consequentemente, impactar positivamente na prevenção de danos à saúde coletiva.

Neste contexto, o presente estudo visou investigar o impacto da AUDIN na manutenção e no aprimoramento do SGQ de uma empresa distribuidora de produtos para saúde localizada em Salvador/BA.

\subsection{O mercado de produtos para saúde e a qualidade}

O mercado de Medical Devices é um dos que mais crescem atualmente no mundo contudo, os gastos com dispositivos médicos no Brasil são baixos e inferiores aos verificados em muitos países, o que indica uma demanda reprimida e um elevado potencial de crescimento (Marrone, 2015).

Por se tratar de um ramo delicado, envolvendo saúde individual e coletiva, tecnologia de ponta e alto impacto econômico, a preocupação com a qualidade é de fundamental importância. Segundo Campos (1992), qualidade é um conjunto de atributos presentes em um produto ou serviço capaz de atender às necessidades do cliente, estando disponível em tempo, forma e lugar certos, por um preço competitivo. Com a globalização e o constante aumento da competitividade, podemos dizer que a padronização das melhores práticas de mercado por meio de sistemas de qualidade bem estruturados acarreta melhor produtividade e redução de custos com retrabalhos, exercendo alto impacto na satisfação dos clientes e gerando um diferencial competitivo importante para a sobrevivência das empresas (Cota \& Freitas, 2013).

Com o objetivo de padronizar a qualidade e tornar os processos auditáveis dentro dos princípios da Qualidade Total, em 1987 a International Standarnization Organization (ISO) publicou a série ISO 9000, que contém a norma ISO 9001, onde são retratados os requisitos básicos para um Sistema de Gestão da Qualidade (Cota \& Freitas, 2013).

A partir dos conceitos e padrões da ISO 9001:2015 (ABNT, 2015) foram desenvolvidas outras normas mais específicas, como a ISO 13.485:2016 (ABNT, 2016), que é a norma internacional da ISO que contém os requisitos para fins regulamentares para os Sistemas de Gestão da Qualidade em empresas do ramo de Dispositivos Médicos. A norma RDC 16/2013 da Agência Nacional de Vigilância Sanitária (ANVISA, 2013) também é uma norma que contém requisitos para as Boas Práticas de Fabricação, Importação e Distribuição de Produtos Médicos e de Diagnóstico In Vitro e possui status de Legislação Sanitária no Brasil, trazendo importantes diretrizes para a confecção, a manutenção e o aprimoramento do SGQ das empresas por ela regulamentadas, dentre os quais, política da qualidade, objetivos, missão, visão e valores, padronização dos processos, controle de documentos, de registros e de mudanças, gestão de riscos, tratativas a reclamações de clientes, não-conformidades e incidentes, planos de ação com adoção de medidas corretivas e preventivas, verificação de eficácia, análise estatística de dados, análise crítica da direção e melhoria contínua.

\subsection{Histórico da regulação sanitária do comércio de produtos para saúde no Brasil e as melhores práticas de medical devices no mundo}

Os marcos regulatórios sobre o comércio de MD no Brasil são a Lei no 5.991 de 17 de dezembro de 1973, que dispõe acerca do controle sanitário sobre o comércio de drogas, medicamentos, insumos farmacêuticos e correlatos e dá outras providências (Brasil, 1973) e a Lei n ${ }^{\circ} 6.360$ de 23 de setembro de 1976, que dispõe sobre a Vigilância Sanitária a que ficam sujeitos os medicamentos, as drogas, os insumos farmacêuticos e correlatos, cosméticos, saneantes e outros produtos e dá outras providências (Brasil, 1976). É importante frisar que durante as décadas de 70 a 90, principalmente na redação das leis e 
regulamentações nacionais, os MD eram chamados de correlatos, conforme definido na Lei $\mathrm{n}^{\circ} 5.991$ de 17 de dezembro de 1973 (Brasil, 1973):

IV - Correlato - a substância, produto, aparelho ou acessório não enquadrado nos conceitos anteriores, cujo uso ou aplicação esteja ligado à defesa e proteção da saúde individual ou coletiva, à higiene pessoal ou de ambientes, ou a fins diagnósticos e analíticos, os cosméticos e perfumes, e, ainda, os produtos dietéticos, óticos, de acústica médica, odontológicos e veterinários. (Brasil, 1973)

Estas duas leis federais da década de 70 são de fundamental importância, pois começaram a normatizar e regular o setor de MD no Brasil, sendo válidas até os dias atuais, com as modificações feitas através de legislações posteriores. Apesar de toda esta relevância histórica, o foco era o setor regulatório, como questões de registros dos produtos no Ministério da Saúde, requisitos para o licenciamento sanitário dos estabelecimentos, requisitos para assunção de responsabilidade técnica, os deveres do responsável técnico e requisitos mínimos para controle de qualidade.

A Garantia da Qualidade, ou Gestão da Qualidade, como conhecemos atualmente, ganhou maior destaque através da RDC 59/2000 (ANVISA, 2000), que ampliou as obrigações legais quanto ao SGQ, dando-lhe uma robustez de requisitos mínimos para garantir as Boas Práticas de Fabricação ou de Distribuição e Armazenamento. Nos anos 2000 a nomenclatura "correlatos" perdeu espaço para "produtos médicos" nas resoluções da Diretoria Colegiada (RDC) da ANVISA, que atualmente tem utilizado frequentemente a nomenclatura "produtos para saúde" em suas publicações. Posteriormente, a RDC 59/2000 foi revogada pela RDC 16/2013 (ANVISA, 2013), que incluiu o gerenciamento de riscos no escopo dos itens obrigatórios no SGQ, ausente na norma anterior.

Atualmente, a norma de referência para as Boas Práticas de Medical Devices no mundo é a ISO 13.485:2016 (ABNT, 2016), que é muito similar à RDC 16/2013 (ANVISA, 2013) e a UE 2017/745 (Parlamento Europeu, 2017), o que demonstra uma convergência internacional no entendimento quanto aos requisitos mínimos para um SGQ de fabricantes, importadores e distribuidores de MD. Segundo o regulamento UE 2017/745 do Parlamento Europeu e do Conselho, de 5 de abril de 2017, relativo aos dispositivos médicos, além de todos os requisitos de conformidade regulatória, há um escopo mínimo para o SGQ: apresentar a estrutura e a documentação do sistema de gestão, incluindo as políticas e os objetivos das suas atividades; definir as políticas de atribuição de atividades e responsabilidades; estabelecer os processos de avaliação e de tomada de decisão em conformidade com os processos, responsabilidades e papel do pessoal e da alta direção; basear-se no ciclo PDCA (planejamento, realização, avaliação e, se necessário, adaptação) para promoção da melhoria contínua do SGQ; estabelecer controle dos documentos e de registos; executar análises críticas da gestão a partir da realização das auditorias internas; estabelecer ações corretivas e preventivas; estabelecer controle de reclamações e notificações de não-conformidade e promover a melhoria contínua (Parlamento Europeu, 2017).

Esta similaridade quanto à regulamentação sanitária em diversos países é imprescindível em um mundo globalizado (Niederländer, Kriza, \& Kolominsky-Rabas, 2017; Parlamento Europeu, 2017), segundo o Comitê Europeu, que apoia que as orientações desenvolvidas para dispositivos médicos ocorram a nível internacional, em particular no contexto da Força-Tarefa de Harmonização Global (GHTF), através de sua iniciativa de acompanhamento, o Fórum Internacional de Reguladores de Dispositivos Médicos (IMDRF), levando em consideração a promoção de uma convergência global de regulamentos. Segundo o Comitê Europeu, esta convergência global nos requisitos de regulamentação contribui para um alto nível de segurança à saúde coletiva em todo o mundo, além de facilitar o comércio, em particular nas disposições sobre Identificação Única de Dispositivo, requisitos gerais de segurança e desempenho, documentação técnica, regras de classificação, procedimentos de avaliação de conformidade e procedimentos clínicos para investigações (Parlamento Europeu, 2017). 
Desta forma, observa-se uma tendência de harmonização global quanto à legislação sanitária, na qual o Brasil já participa ativamente. Não podemos nos esquecer que uma das propostas explícitas da RDC 16/2013 foi de promover uma harmonização legal quanto ao Mercosul (ANVISA, 2013) e diversas ações têm sido tomadas para uma harmonização global.

\subsection{Auditorias internas na promoção da melhoria contínua em sistemas de gestão da qualidade}

As auditorias internas da qualidade são ferramentas essenciais para verificação da conformidade do SGQ (Levin, 2001; Costa, et al., 2006). Através delas, é possível encontrar possíveis não-conformidades (NC) e oportunidades de melhoria (OM). Os relatórios das auditorias internas devem ser "entradas" nos processos de análise crítica da direção, cujas "saídas" correspondentes devem estar em um plano de ação para implementação das OM, com análise crítica da causa-raiz das NC, medidas corretivas, preventivas e posterior avaliação de eficácia das medidas adotadas, promovendo, deste modo, uma checagem regular do SGQ e sua melhoria contínua, seguindo o fluxo do Ciclo PDCA (Paladini, 2004).

A RDC 16/2013 define auditorias da qualidade como o estabelecimento e a padronização de um exame sistemático e independente do SGQ, que deve levar em consideração regularidade e frequência suficientes para assegurar a satisfação das especificações e padronizações estabelecidas, de modo que procedimentos sejam implementados eficientemente e que sejam adequados para alcançar os objetivos do SGQ (ANVISA, 2013), o que está de acordo com as boas práticas internacionais, sendo similarmente descrito na ISO 9001:2015 (ABNT, 2015), na ISO 13.485:2016 (ABNT, 2016) e na UE 2017/745 (ABNT, 2015; ABNT, 2016; Parlamento Europeu, 2017), ressaltando-se seu aspecto essencial para a resolução e a prevenção de desvios de qualidade, mitigação de riscos e de possíveis danos à saúde individual e coletiva (Costa, et al., 2006).

\section{Metodologia}

A pesquisa foi realizada através de um estudo de caso, seguindo metodologia exploratória descritiva e explicativa, de abordagem qualitativa e quantitativa, durante os meses de janeiro e fevereiro de 2021. Foi utilizada como referencial metodológico a norma NBR ISO 19011:2018 (ABNT, 2018), que fornece diretrizes para auditorias de sistemas de gestão e as recomendações metodológicas para pesquisa exploratória de Gil (2010). Os dados coletados foram referentes ao SGQ de uma empresa importadora e distribuidora de produtos para saúde, localizada na cidade de Salvador/BA, que comercializa produtos para saúde fabricados no sudeste asiático, Europa, Estados Unidos e Brasil, de Classe de Risco I, II III e IV, segundo classificação da ANVISA (ANVISA, 2011). A fim de organizar a coleta e a análise dos dados, a pesquisa foi realizada em três etapas: reunião de abertura, observação in loco e análise dos dados e relatórios.

A primeira etapa contemplou uma reunião de abertura, explicando a proposta da pesquisa, com a presença dos diretores e líderes, seguida da aplicação do check-list de auditoria interna, preenchido com as informações obtidas durante a reunião, bem como com os dados obtidos nas etapas posteriores. A reunião de abertura contou com a participação de seis líderes da empresa: gerente geral, gerente de recursos humanos, coordenador de estoque e logística, responsável técnico, diretor administrativo e diretor comercial. O check-list de auditoria interna utilizado foi criado como uma adaptação dos requisitos da RDC 16/2013 e outras legislações pertinentes ao setor à realidade da empresa e seu escopo funcional (ANVISA, 2013).

A segunda etapa foi de observação direta in loco, a fim de evidenciar a compatibilidade entre os documentos do SGQ e a rotina diária dos fluxos, processos, atividades e registros. Foi realizado acompanhamento de campo nas atividades de outros oito colaboradores, contemplando todos os setores da organização: um estoquista, um motorista, um instrumentador cirúrgico, um diretor e um auxiliar administrativo de cada um dos setores de cotação, faturamento, recursos humanos e qualidade. 
A terceira etapa foi a análise dos dados obtidos nas duas etapas anteriores, com confecção de um relatório da auditoria interna e de um plano de ação, com medidas corretivas e preventivas relativas às não-conformidades e oportunidades de melhoria, a ser realizada conjuntamente com diretores e gestores da "empresa caso", conforme estabelecido no Quadro 1:

Quadro 1: Instrumentos de coleta de dados.

\begin{tabular}{|l|c|c|}
\hline Instrumento de coleta & Universo pesquisado & Finalidade \\
\hline Reunião e questionário & governança e SGQ & verificar a conformidade do SGQ \\
\hline Observação direta & fluxos e processos in loco & verificar a compatibilidade com SGQ \\
\hline Documentos & todos os que compõem o SGQ & evidenciar conformidade do SGQ com a norma \\
\hline Dados arquivados & registros e rastreabilidade & comprovar a rastreabilidade e os registros \\
\hline
\end{tabular}

Fonte: Autores.

Ao final, foi realizada a verificação da eficácia das ações na correção das não-conformidades elencadas.

\section{Resultados e Discussão}

Os resultados obtidos com a realização da auditoria interna, baseada na RDC 16/2013 e apoiada pela ISO 13.485:2016, com 56 tópicos de verificação de conformidade, adaptados para a realidade da "empresa caso", conforme ajuste de escopo previsto pela RDC 16/2013, estão expressos no Quadro 2.

Quadro 2: Respostas ao Questionário de Auditoria Interna de verificação de conformidade com a norma RDC 16/2013 aplicado à realidade da "empresa caso".

\begin{tabular}{|c|c|c|c|c|}
\hline Requisitos - RDC 16/2013 & Evidências & $\mathbf{C}$ & NC & $\mathbf{O M}$ \\
\hline 1. A empresa mantém um SGQ? (item 2.1) & Apresentados documentos do SGQ & $\mathrm{X}$ & & \\
\hline 2. Documentos acessíveis aos usuários? (item 2.2.1) & Acessibilidade via intranet & $\mathrm{X}$ & & \\
\hline 3. Política da Qualidade estabelecida? (item 2.2.1) & No Manual da Qualidade e treinamentos & $\mathrm{X}$ & & \\
\hline 4. POP de Auditorias da Qualidade? (item 7.3.1) & POP-016 e RQ 08 & $\mathrm{X}$ & & \\
\hline 5. Registros das auditorias e análise? (item 7.3.2; 7.3.3) & RQ 08 e RQ 14 & $X$ & & \\
\hline 6. Manutenção de equipamentos médicos? (item 5.1.5.1) & POP 023 e RQ 13 & $\mathrm{X}$ & & \\
\hline 7. Há registros e laudos das manutenções? (item 5.1.5.1) & RQ 13 & $\mathrm{X}$ & & \\
\hline $\begin{array}{l}\text { 8.Sistemática para Reclamação de clientes (item } 7.1 \text { e } \\
7.2 \text { ) }\end{array}$ & POP 015 & $X$ & & \\
\hline 9. Registro de medidas corretivas e preventivas? (7.1.) & RQ 07 e NC 011220 & $X$ & & \\
\hline 10.QT para detentor do registro nas falhas? (item 7.2) & NC 010620 da QT de material danificado & $\mathrm{X}$ & & \\
\hline 11. Tratativa de NC de qualidade e recall (item 7.1.1.8) & POP 007 & $\mathrm{X}$ & & \\
\hline 12. Suspeita de NC de lote e recall (item 7.1.1.8) & POP 007 & $\mathrm{X}$ & & \\
\hline 13. Área para produtos NC (item 6.5.1.) & inspeção in loco na área de segregação & $\mathrm{X}$ & & \\
\hline 14. Destinação para os produtos NC (item 6.5) & rastreabilidade e registros & $\mathrm{X}$ & & \\
\hline 15. Potabilidade da água (item 5.1.4) & laudos & $\mathrm{X}$ & & \\
\hline 16. Troca periódica dos filtros (item 5.1.4) & RQ 10 e inspeção in loco & $\mathrm{X}$ & & \\
\hline 17. controle de pragas (item 5.1.3.4) & RQ 05 e certificados & $\mathrm{X}$ & & \\
\hline
\end{tabular}




\begin{tabular}{|c|c|c|c|}
\hline 18. Controle de temperatura (item 5.1.3) & POP 10 e registros & $\mathrm{X}$ & \\
\hline 19. Gerenciamento de risco (item 2.4) & Programa de Gerenciamento de Riscos & $\mathrm{X}$ & \\
\hline 20. Gerenciamento de resíduos (item 5.1.3.5) & PGRSS, contrato e certificados & $\mathrm{X}$ & \\
\hline 21. Os documentos regulatórios válidos & Apresentados Licenças, AFE, CRT & $\mathrm{X}$ & \\
\hline 22. Controle de documentos (itens 3.1.1 a 3.1.6.) & POP 001 e lista mestra & $\mathrm{X}$ & \\
\hline 23. Assinatura nos documentos (item 3.1.2.) & nomes definidos, mas não assinado & & $\mathrm{X}$ \\
\hline 24.Calibração (item 5.4.1) & encontrado um laudo vencido & & $\mathrm{X}$ \\
\hline 25. Análise estatística de desempenho (item 9.2) & apresentados documentos & $\mathrm{X}$ & \\
\hline 26.Definição do representante (item 2.2.5) & apresentado comunicado & $\mathrm{X}$ & \\
\hline 27.Análises Críticas e Revisões Gerenciais (item 2.2.6) & há atas não localizadas & & $\mathrm{X}$ \\
\hline 28. Adequação das instalações (item 5.1.2) & inspeção in loco & $\mathrm{X}$ & \\
\hline 29. Instalações limpas e identificadas? (item 5.1.3.1.) & RQ 04 inspeção in loco & $\mathrm{X}$ & \\
\hline 30. POP de limpeza (item 5.1.3.1) & POP 018 e RQ 04 & $X$ & \\
\hline 31. POP transporte (item 6.1.1) & POP 006 e licença dos veículos & $\mathrm{X}$ & \\
\hline 32.Higienização dos veículos (item 5.1.3.1.) & RQ 05, RQ 11 e RQ 15 & $\mathrm{X}$ & \\
\hline 33. Mecanismos anti-incêndio (item 2.4.1) & apresentado AVCB & $\mathrm{X}$ & \\
\hline 34. Organograma (item 2.2.2) & apresentado o organograma & $\mathrm{X}$ & \\
\hline $\begin{array}{l}\text { 35. DCC com responsabilidades e autoridades (item } \\
\text { 2.3.1) }\end{array}$ & apresentados documentos & $\mathrm{X}$ & \\
\hline 36. Treinamento (item 2.3.2) & apresentados registros de treinamento & $\mathrm{X}$ & \\
\hline 37. PPRA, PCMSO e ASO (item 5.1.4; NR-7 E NR-9) & encontrados alguns ASO vencidos & & $\mathrm{X}$ \\
\hline 38. Uso de EPI (item 5.1.4) & verificado in loco & $\mathrm{X}$ & \\
\hline 39.Rastreabilidade dos processos (itens 6.3 e 6.4 ) & POP, sistema informatizado e registros & $\mathrm{X}$ & \\
\hline 40. Há registros rastreáveis completos (itens 6.3 e 6.4) & sem lotes pontuais de material de apoio & & $\mathrm{X}$ \\
\hline 41. Rastreabilidade de utilização nas Nfe (6.3 e 6.4) & verificado em documentos & $\mathrm{X}$ & \\
\hline 42. Controle de Compras (itens $2.5 .1 ; 2.5 .4$ ) & POP 008 e pedidos de compras & $\mathrm{X}$ & \\
\hline 43. Conformidade compra $\mathrm{x}$ chegada $(2.5 .1)$ & POP 004 e registros & $\mathrm{X}$ & \\
\hline 44. Qualificação dos fornecedores (item 2.5.3) & POP 009 e RQ 001 & $\mathrm{X}$ & \\
\hline 45. Controle de terceirização (item 2.5.2.) & apresentados contratos e registros & $\mathrm{X}$ & \\
\hline 46. Controle sobre fornecedores (item 2.5.2) & RQ 001 & $\mathrm{X}$ & \\
\hline 47. Inspeção de recebimento (itens 5.3.1; 6.1.3) & POP 004 e registros & $\mathrm{X}$ & \\
\hline $\begin{array}{l}\text { 48. Integridade de rótulos e instruções de uso (item } \\
5.2 .2 .1 \text { ) }\end{array}$ & POP 004, registros e inspeção in loco & $\mathrm{X}$ & \\
\hline 49. Método para evitar mistura acidental (item 5.2.2.1) & POP 004 e inspeção in loco & $\mathrm{X}$ & \\
\hline 50. Legibilidade dos rótulos (item 5.2.2.2) & POP 004 e inspeção in loco & $\mathrm{X}$ & \\
\hline 51. Inspeção dos rótulos (item 5.2.2.3) & POP 004 e registros & $\mathrm{X}$ & \\
\hline $\begin{array}{l}\text { 52. Separação do de recebimento e expedição (item } \\
\text { 5.1.2) }\end{array}$ & visualização in loco & $\mathrm{X}$ & \\
\hline 53. Identificação das áreas (item 5.1.2) & visualização in loco & $\mathrm{X}$ & \\
\hline 54. POP recebimento de materiais/produtos (item 2.5.1) & POP 004 e registros & $\mathrm{X}$ & \\
\hline 55. Identificação de materiais (item 2.5.1) & POP 004 e registros & $\mathrm{X}$ & \\
\hline 56. POP para armazenagem (item 6.2.1) & POP 004 & $\mathrm{X}$ & \\
\hline
\end{tabular}

Fonte: Dados da pesquisa.

Apesar de não terem sido encontradas não-conformidades gerais, no sentido de não atendimento de um item da norma por completo, a localização de uma NC pontual durante a inspeção foi considerada como NC para todo o requisito. Isto foi 
feito, por exemplo, na falta de rastreabilidade de lotes amostrais de um instrumental cirúrgico (item 40 do check-list), o que foi sinalizado como NC, independentemente da apresentação de evidências de conformidade para o requisito na rastreabilidade dos demais produtos. Esta atitude visou garantir uma revisão sistemática do tópico onde a NC foi encontrada, a fim de permitir uma melhoria no controle da qualidade para aquele requisito, seja no procedimento padrão, nos registros ou no treinamento de colaboradores responsáveis pela excecução. Este tipo de condução é extremamente importante, principalmente no que tange à rastreabilidade, pois estreita a vigilância necessária para garantir a segurança dos pacientes e uma possível identificação em casos de recall ou descobertas de problemas relacionados ao lote de algum produto, bem como permite um rastro para notificações de vigilância em saúde, tendo pleno controle da utilização dos itens em todo seu ciclo de vida (Lovis, 2018; Tarricone, et al., 2021).

Não foram encontradas NC relativas à falta de documentos, documentos em versão desatualizada ou fora do período estabelecido para revisão, ou NC referentes à descrição de processos incompatíveis com a RDC 16/2013 nos documentos que compõem o SGQ da "empresa caso". A lista dos documentos do SGQ analisados estão presentes no Quadro 3.

Quadro 3: Relação dos documentos do SGQ da "empresa caso" avaliados na auditoria interna.

\begin{tabular}{|c|c|}
\hline Código & Descrição do Documento \\
\hline MQ & Manual da Qualidade \\
\hline PGR & Programa de Gerenciamento de Riscos \\
\hline PGRSS & Programa de Gerenciamento de Resíduos em Serviços de Saúde \\
\hline POP-001 & Controle de Documentos, Dados e Registros \\
\hline POP-002 & Comunicação \\
\hline POP-003 & Análise Crítica da Direção \\
\hline POP-004 & Inspeção de Entrada, Controle de Rotulagem e Armazenamento \\
\hline POP-005 & Saída de Materiais \\
\hline POP-006 & Transporte de Materiais \\
\hline POP-007 & Identificação e Rastreabilidade com Plano de Contingência \\
\hline POP-008 & Controle de Compras \\
\hline POP-009 & Qualificação de Fornecedores e Prestadores de Serviço \\
\hline POP-010 & Controle de Temperatura do Estoque \\
\hline POP-011 & Controle de Mudanças \\
\hline POP-012 & Controle de Materiais a Vencer \\
\hline POP-013 & Retorno de Materiais Enviados para Cirurgias \\
\hline POP-014 & Componentes e Produtos Não Conformes \\
\hline POP-015 & Tratativa de Não-Conformidades e Análise Estatística \\
\hline POP-016 & Auditoria Interna \\
\hline POP-017 & Saída de Materiais para Esterilização \\
\hline POP-018 & Limpeza das Áreas de Trabalho \\
\hline POP-019 & Cotação \\
\hline POP-020 & Faturamento \\
\hline POP-021 & Responsabilidade Técnica e Regulatório \\
\hline POP-022 & Capacitação, Treinamento e Conscientização \\
\hline POP-023 & Manutenção Preventiva e Corretiva de Equipamentos \\
\hline RQ 01 & Lista de Fornecedores e Prestadores de Serviço Aprovados \\
\hline RQ 02 & Registro de Avaliação e Qualificação de Fornecedores \\
\hline RQ 03 & Controle de Temperatura \\
\hline
\end{tabular}




\begin{tabular}{|c|c|}
\hline RQ 04 & Relatório de Limpeza das Áreas de Trabalho \\
\hline RQ 05 & Planilha de Controle de Desinsetização \\
\hline RQ 06 & Formulário de Notificação de Material Danificado \\
\hline RQ 07 & Registro de Não-Conformidade e Reclamação de Cliente \\
\hline RQ 08 & Check-list de Auditoria Interna \\
\hline RQ 09 & Pesquisa de Satisfação de Clientes \\
\hline RQ 10 & Planilha de Controle dos Filtros de Água \\
\hline RQ 11 & Planilha de Controle de Higieização dos Veículos de Transporte \\
\hline RQ 12 & Lista de Presença em Evento \\
\hline RQ 13 & Controle de Manutenção Preventiva \\
\hline RQ 14 & Ata de Análise Crítica da Direção \\
\hline RQ 15 & Planilha de Controle de Manutenção dos Veículos \\
\hline RQ 16 & Avaliação de Desempenho - SWOT Pessoal \\
\hline DCC01 & Descrição de Cargos e Competências - Responsável Técnico \\
\hline DCC02 & Descrição de Cargos e Competências - Coordenador de Estoque \\
\hline $\mathrm{DCC} 03$ & Descrição de Cargos e Competências - Estoquista \\
\hline DCC04 & Descrição de Cargos e Competências - Motorista Entregador \\
\hline DCC05 & Descrição de Cargos e Competências - Instrumentador Cirúrgico \\
\hline DCC06 & Descrição de Cargos e Competências - Gerente Administrativo \\
\hline DCC07 & Descrição de Cargos e Competências - Auxiliar ADM Cotação \\
\hline DCC08 & Descrição de Cargos e Competências - Auxiliar ADM Faturamento \\
\hline DCC09 & Descrição de Cargos e Competências - Gerente de Recursos Humanos \\
\hline DCC10 & Descrição de Cargos e Competências - Promotor de Vendas \\
\hline DCC10 & Descrição de Cargos e Competências - Compliance Officer \\
\hline DCC12 & Descrição de Cargos e Competências - DPO \\
\hline MT01 & Matriz de Treinamento - Responsável Técnico \\
\hline MT02 & Matriz de Treinamento - Coordenador de Estoque \\
\hline MT03 & Matriz de Treinamento - Estoquista \\
\hline MT04 & Matriz de Treinamento - Motorista Entregador \\
\hline MT05 & Matriz de Treinamento - Instrumentador Cirúrgico \\
\hline MT06 & Matriz de Treinamento - Gerente Administrativo \\
\hline MT07 & Matriz de Treinamento - Auxiliar ADM Cotação \\
\hline MT08 & Matriz de Treinamento - Auxiliar ADM Faturamento \\
\hline MT09 & Matriz de Treinamento - Gerente de Recursos Humanos \\
\hline MT10 & Matriz de Treinamento - Promotor de Vendas \\
\hline MT11 & Matriz de Treinamento - Compliance Officer \\
\hline MT12 & Matriz de Treinamento - DPO \\
\hline
\end{tabular}

Fonte: Dados da pesquisa.

A existência de documentos definindo políticas da qualidade e padronização das atividades, principalmente quando pautadas em requisitos claros e internacionalmente reconhecidos, apoiados por registros bem estruturados, são considerados como a base do processo regulatório (Niederländer, \& Kriza, \& Kolominsky-Rabas, 2017) e sua análise deve ser criteriosamente realizada, pautando não só na adequação, mas também nas oportunidades de melhoria para os processos e a gestão da qualidade (ANVISA, 2013; ABNT, 2015; ABNT, 2016; Niederländer, \& Kriza, \& Kolominsky-Rabas, 2017). 
Com relação a registros, não foi encontrada a ata da última análise crítica da direção (item 27 do check-list), apesar de terem sido apresentados e-mails com as saídas da reunião, definindo medidas corretivas e preventivas relacionadas aos dados de entrada apresentados. Também haviam registros fora do escopo de validade, como alguns atestados de saúde ocupacional (ASO) e o laudo de calibração de um dos termohigrômetros. A falta de registros apresenta uma falha no seguimento das boas práticas de governança corporativa, principalmente no que tange à manutenção de dados do histórico do desenvolvimento do sistema de gestão da qualidade, suas falhas e possibilidades de aprimoramento (Brown, 2019).

Seguindo-se os princípios da melhoria contínua, os resultados da auditoria interna serviram de entradas para a reunião de análise crítica e dela se originou um plano de ação, com medidas corretivas, preventivas e verificação de eficácia, devidamente organizadas em cronograma com definição de responsabilidades, "girando" o ciclo PDCA da empresa (Paladini, 2004; Gonzales \& Martins 2011; Segalote \& Neto, 2016; Brown, 2019).

O Gráfico 1 apresenta o progresso de implementação das melhorias e o aprimoramento do SGQ da empresa frente aos problemas encontrados, através de verificação de eficácia das ações corretivas.

Gráfico 1: Status de verificação de eficácia do plano de ação para correção das NC listadas no relatório de auditoria interna, após sete e 20 dias de apresentação.

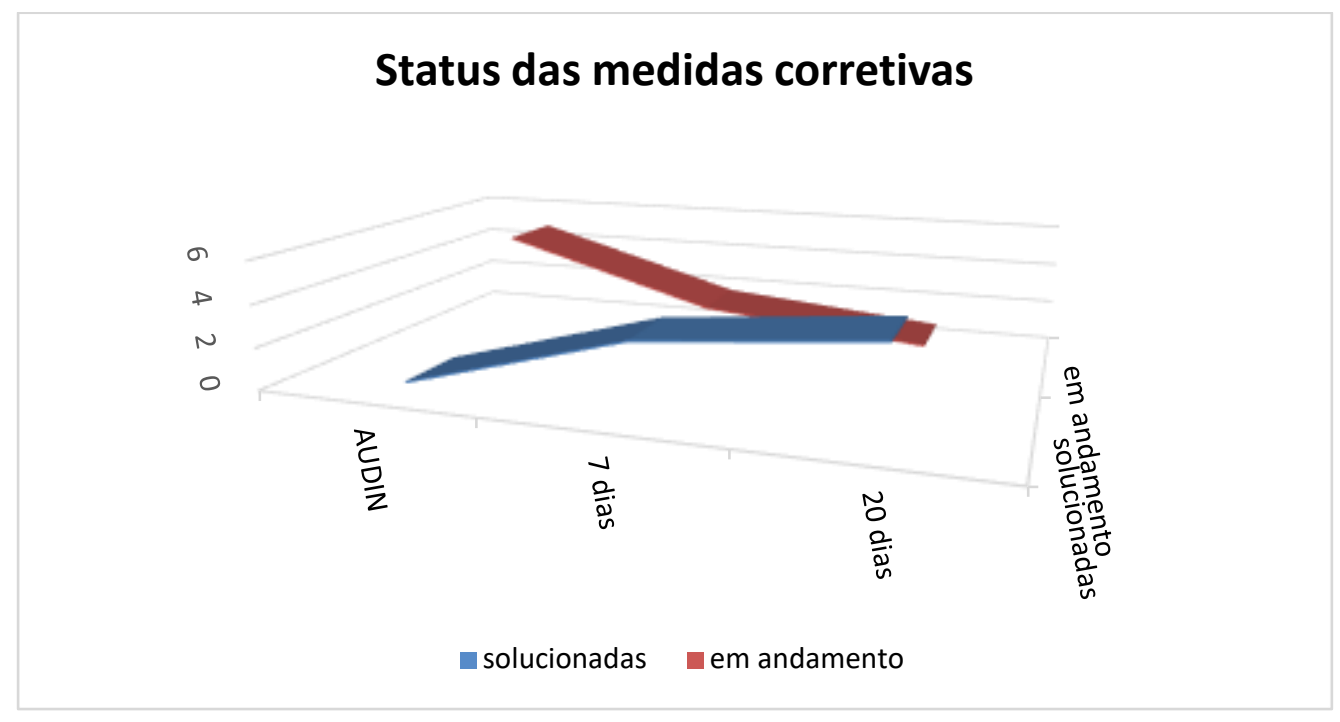

Fonte: Autores.

Conforme observado no Gráfico 1, após sete dias de implementação do plano de ação, $60 \%$ das NC estavam solucionadas e $40 \%$ estavam com as devidas medidas corretivas em andamento e, após 20 dias, $80 \%$ das NC estavam solucionadas e $20 \%$ ainda estavam com medidas corretivas em andamento.

Foi constatado que a "empresa caso" se baseou na RDC 16/2013 e na ISO 13.485:2016 para confeccionar sua última revisão dos documentos do SGQ, ocorrida em 2020. A padronização das atividades, etapa base para criação de um SGQ estava bem estabelecida no Manual da Qualidade, em programas e políticas, nos 23 procedimentos operacionais padronizados (POP), nos registros e nas instruções de trabalho. Todos os aspectos considerados essenciais pela UE 2017/745 estavam estabelecidos (Parlamento Europeu, 2017) e havia registros das atividades críticas, conforme recomendado na literatura e nas melhores práticas de mercado (ANVISA, 2013; ABNT, 2016; Niederländer, Kriza \& Kolominsky-Rabas, 2017).

Observou-se que "empresa caso" baseia-se principalmente no Ciclo PDCA, para promoção da melhoria contínua, tendo as reclamações de clientes, os relatórios de $\mathrm{NC}$ e os resultados das auditorias internas como fonte de alimentação para o ciclo. Há ainda uma integração parcial com o modelo 5S no layout e nas rotinas setoriais. O ciclo PDCA é uma das principais 
ferramentas da gestão da qualidade para verificar falhas reais ou possíveis e estabelecer melhorias o SGQ, servindo de modelo para outras, como o método de análise e solução de problemas (MASP) (Paladini, 2004; Gonzales \& Martins, 2011; ANVISA, 2013; Segalote \& Neto, 2016).

A auditoria interna da qualidade foi utilizada como ferramenta essencial para verificação da conformidade do SGQ, conforme definido na literatura e nas melhoras práticas de mercado (Levin, 2001; ANVISA, 2013; ABNT, 2015; ABNT, 2016; Parlamento Europeu, 2017). Seu relatório foi documento de entrada em processo de análise crítica da direção, originando como saída, um plano de ação para implementação das medidas corretivas e preventivas, com organograma e definição dos responsáveis por cada atividade, além de avaliação de eficácia das medidas adotadas, promovendo, deste modo, uma checagem regular do SGQ e acompanhamento de sua melhoria, atendendo ao requisito da RDC 16/2013 e, similarmente, ao que é descrito na ISO 9001:2015 (ABNT, 2015), na ISO 13.485:2016 (ABNT, 2016) e na (UE) 2017/745 (Parlamento Europeu, 2017). O monitoramento do cumprimento do plano de ação apresentou eficácia de resolução de $60 \%$ das NC em sete dias e de $80 \%$ em vinte dias.

\section{Considerações Finais}

A RDC 16/2013 e a ISO 13.485:2016 foram as fontes referenciais utilizadas na criação do SGQ da "empresa caso", servindo de base para sua confecção, manutenção e aprimoramento do mesmo, ressaltando que a adequação à RDC 16/2013 é requisito legal para funcionamento de uma distribuidora de produtos para saúde no Brasil. Um dos principais desafios na utilização destas normas para confecção de um SGQ é definir o escopo correto, desconsiderando os itens da norma que não são pertinentes às atividades executadas pela empresa. Este tipo de dificuldade metodológica é particularmente comum quando normas são criadas de modo a abranger um grande número de empresas com diferentes perfis, como, neste caso, fabricantes, importadores e distribuidores. A definição de um escopo de exclusões de itens não pertinentes e suas justificativas carece de uma discussão mais aprofundada.

A auditoria interna demonstrou ser uma ferramenta eficaz para a manutenção e o aprimoramento do SGQ da "empresa caso", pois utiliza as não-conformidades identificadas para confecção de planos de ação, objetivando melhoria contínua, com alto índice de eficácia em curtos espaços de tempo (60\% em sete dias e $80 \%$ em vinte dias), entretanto, estudos similares precisam ser realizados para que obtenção de dados mais robustos e comparação de resultados, a fim de compreender melhor como esta ferramenta se comporta para o setor pesquisado.

Destaca-se que a realização periódica das auditorias internas é extremamente importante, pois permite verificar a eficácia das medidas corretivas e preventivas a longo prazo, bem como a adesão à cultura da qualidade e aos procedimentos definidos pela organização, essenciais para garantir que não haja impacto negativo à saúde dos pacientes atendidos com os produtos fornecidos, bem como à saúde coletiva em geral. Deste modo, estudos de acompanhamento a longo prazo também são necessários.

\section{Referências}

Agência Nacional de Vigilância Sanitária (ANVISA) (2000). Resolução da Diretoria Colegiada - RDC $n^{o}$ 59, 29/06/2000. Diário Oficial da União, Brasília.

Agência Nacional de Vigilância Sanitária (ANVISA) (2001). Resolução da Diretoria Colegiada - RDC $n^{o}$ 185, 22/10/2001. Diário Oficial da União, Brasília.

Agência Nacional de Vigilância Sanitária (ANVISA) (2011). Resolução da Diretoria Colegiada - RDC $n^{\circ}$ 61, 18/11/2011. Diário Oficial da União, Brasília.

Agência Nacional de Vigilância Sanitária (ANVISA) (2013). Resolução da Diretoria Colegiada - RDC $n^{o} 16,28 / 03 / 2013$. Diário Oficial da União, Brasília. 
Associação Brasileira de Normas Técnicas (2015). ABNT NBR ISO 9001:2015: Sistemas de gestão da qualidade - requisitos.

Associação Brasileira de Normas Técnicas (2016). ABNT NBR ISO 13.485:2016: Produtos para Saúde - Sistemas de gestão da qualidade - Requisitos para fins Regulamentares. Rio de Janeiro.

Associação Brasileira de Normas Técnicas (2018). ABNT NBR ISO 19011:2018: Diretrizes para Auditoria de Sistemas de Gestão. Rio de Janeiro.

Brasil (1973). Lei $n^{\circ}$ 5.991, 17/12/1973. Diário Oficial da União, Brasília.

Brasil (1976). Lei $n^{\circ} 6.360,23 / 07 / 1976$. Diário Oficial da União, Brasília.

Costa, C. F., Rambo, D. A., Medeiros, I. B. de O., Corso, K. B., \& Regauer, T. (2006). Auditoria Interna das normas de qualidade para obtenção da ISO: um estudo de caso na indústria têxtil Dudalina S/A. In: III Simpósio de Excelência em Gestão e Tecnologia; Rezende, Rio de Janeiro.

Cota, K. A., \& Freitas, M. A. M. (2013). Gestão da Qualidade, um desafio permanente: um estudo de caso sobre o processo de manutenção de um sistema de qualidade em uma indústria metalúrgica. Produto \& Produção, 4(2), 59-71. https://doi.org/10.22456/1983-8026.31756

Gil, A. C. (2010). Atlas metodologia do ensino superior. São Paulo: Atlas.

Guerra-Bretaña, R. M., \& Florez-Rendón, A. L. (2018). Impact of regulations on innovation in the field of medical devices. Res. Biomed. Eng., 34(4), 356-367. https://doi.org/10.1590/2446-4740.180054

Levin, M. (2001). More than standards and regulations are needed to provide safe and effective devices. Biomed Instrum Technol, 35(5), 331-7.

Lingg, M. et al. (2018). Strategies to improve the medical devices life cycle in Mexico. Salud pública Méx., 60(4), 462-471. https://doi.org/10.21149/9060

Martins, A. I. M. (2013). Sistema de Gestão da Qualidade numa Indústria de Dispositivos Médicos: O caso da Plux, Wireless Biosignals, SA. 2013. 87f. [Dissertação]. Lisboa: Pós-graduação em Engenharia e Gestão Industrial, Universidade de Lisboa.

Marrone, P. V. (2015). Saúde 4.0: Propostas para Impulsionar o Ciclo de Inovações em Dispositivos Médicos (DMAs) no Brasil. ABIIS.

Niederländer, C. S., \& Kriza C., \& Kolominsky-Rabas P. (2017). Quality criteria for medical device registries: best practice approaches for improving patient safety-a systematic review of international experiences. Expert Rev Med Devices, 14(1), 49-64. https://doi.org/10.1080/17434440.2017.1268911

Paladini, E. P. (2004). Gestão da Qualidade - Teoria e Prática. São Paulo: Editora Atlas.

Parlamento Europeu. Comitê Europeu (CE). (2017). Regulamento (UE) 2017/745 relativo aos dispositivos médicos. E-OJ, L 117, 1-175.

Lovis, C. (2008). Traceability in healthcare: crossing boundaries. Yearb Med Inform. 105-13.

Tarricone, R., et al. (2021). Establishing a national HTA program for medical devices in Italy: Overhauling a fragmented system to ensure value and equal access to new medical technologies. Health Policy, 125(5), 602-608. https://doi.org/10.1016/j.healthpol.2021.03.003

Brown, A. (2019). Understanding corporate governance of healthcare quality: a comparative case study of eight Australian public hospitals. BMC Health Serv Res., 19(1), 725. https://doi.org/10.1186/s12913-019-4593-0

Gonzales, R. V. D., \& Martins, M. F. (2011). Melhoria contínua e aprendizagem organizacional: múltiplos casos em empresas do setor automobilístico. Gest. Prod., 18(3), 473-486.

Santos, O. S., et al., (2020). Processo de melhoria contínua: estudo de caso aplicado em uma empresa gráfica. Research, Society an Development, 9(9), 1-21. http://dx.doi.org/10.33448/rsd-v9i9.7204

Segalote, F., \& Neto, J. V. (2016). MASP e Ferramentas da Qualidade na Melhoria de Processos em uma Instituição Pública Federal. In: XII Congresso Nacional de Excelência em Gestão \& III INOVARSE - Responsabilidade Social Aplicada. 